\title{
Proposição e análise de um sistema de gerenciamento ativo de tráfego em um estudo de caso de uma autoestrada brasileira
}

\author{
Felipe Caleffi ${ }^{1}$ e Helena Beatriz Bettella Cybis ${ }^{2}$
}

\begin{abstract}
Resumo: O objetivo principal deste artigo é implementar e testar estratégias de gerenciamento ativo de tráfego em um trecho de uma autoestrada brasileira, a fim de avaliar e quantificar a eficácia destas estratégias. O gerenciamento ativo de tráfego é um sistema que gerencia dinamicamente o fluxo com base nas condições de tráfego existentes. O software de microssimulação de tráfego usado para modelagem foi o VISSIM, e foi calibrado para representar as particularidades do comportamento dos motoristas na autoestrada em estudo. As estratégias empregadas na simulação foram a de harmonização da velocidade e a do uso temporário do acostamento. O estudo demonstrou que o gerenciamento ativo de tráfego tem impactos positivos na operação do tráfego e os benefícios mensurados na simulação incluem: redução de headways, redução nos tempos médios de viagem e na variação dos tempos de viagem, e redução no número de trocas de faixa. O uso das estratégias também trouxe redução do tempo de congestionamento, aumentando a eficiência do trecho.

Palavras-chave: Gerenciamento ativo de tráfego, Harmonização da velocidade, Uso temporário do acostamento, Vissim.
\end{abstract}

\begin{abstract}
The main objective of this paper is to implement and test active traffic management (ATM) strategies on a Brazilian freeway segment in order to assess and quantify the effectiveness of these strategies. Active traffic management dynamically manages traffic flow based on existing traffic conditions. Simulation software VISSIM was used to evaluate the simulated ATM strategies, and was calibrated to reflect the freeway driver's behavior. Simulated ATM strategies were speed harmonization and temporary use of hard shoulders. The results showed that active traffic management has a positive impact on traffic operation and benefits of the modeled strategies included: shorter headways, reduction in travel times, reduction in travel time variability and reduction in number of lane changes. Tested strategies also reduced the duration of congestion, increasing the efficiency of the segment under analysis.

Keywords: Active traffic management, Speed harmonization, Temporary use of hard shoulder, Vissim.
\end{abstract}

\section{INTRODUÇÃO}

Técnicas de gerenciamento de tráfego apoiadas em sistemas inteligentes de transporte vêm sendo utilizadas em vários países com o objetivo de reduzir os impactos negativos dos congestionamentos em rodovias. Através de sistemas integrados e respostas coordenadas, o gerenciamento ativo de tráfego pode maximizar a segurança e fluidez do fluxo, e dinamicamente gerir e controlar o tráfego com base nas condições de tráfego existentes. Esta abordagem consiste em uma combinação de estratégias operacionais que ajudam a aperfeiçoar a operação da infraestrutura existente e oferecem benefícios mensuráveis para a rede de transporte.

Os objetivos principais do gerenciamento ativo de tráfego são (Mirshahi et al, 2007): reduzir o congestionamento; reduzir a variação das velocidades dos veículos; aumentar a capacidade da rodovia; tornar o tempo de viagem mais confiável; melhorar a segurança, reduzindo o número e a gravidade dos incidentes; reduzir o tempo de resposta em acidentes; e transmitir um maior número de informações aos usuários, reduzindo, consequente-

\footnotetext{
Felipe Caleffi, Laboratório de Sistema de Transportes - LASTRAN, Universidade Federal do Rio Grande do Sul (f.caleffi@yahoo.com).

${ }^{2}$ Helena Beatriz Bettella Cybis, Laboratório de Sistema de Transportes LASTRAN, Universidade Federal do Rio Grande do Sul (helenabc@producao.ufrgs.br).
}

Manuscrito recebido em 20/05/2015 e aprovado para publicação em 25/02/2016.

Este artigo é parte de TRANSPORTES v. 24, n. 1, 2016 ISSN: 2237-1346 (online). DOI: 10.14295/transportes.v24i1.925 mente, o stress dos motoristas. Avaliações dos impactos de implantação do gerenciamento ativo de tráfego podem ser encontradas em Mirshahi et al. (2007), Carlson et al. (2010), Geistefeldt (2012) e Vadde et al. (2012).

As principais estratégias do gerenciamento ativo de tráfego incluem a harmonização da velocidade e o uso temporário do acostamento. Harmonização da velocidade envolve o uso de limites de velocidade variáveis (LVV) para reduzir os limites de velocidade da via em áreas de congestionamento, e assim manter um melhor fluxo do tráfego e reduzir o risco de colisões. O uso temporário do acostamento tem o objetivo de fornecer uma faixa de rolamento adicional para aumentar a capacidade da rodovia durante um congestionamento ou em períodos que as vias estão sobrecarregadas, e é geralmente implantado em conjunto com harmonização da velocidade para reduzir as velocidades praticadas antes da liberação do uso do acostamento (Sisiopiku et al., 2009).

No Brasil, estratégias de harmonização da velocidade combinadas com o uso temporário do acostamento não são empregadas. O comportamento da corrente de tráfego observado no trecho em estudo, e frequentemente observado em outras autoestradas brasileiras faz do país um bom candidato a receber estas estratégias inovadoras. Como apontado por Caleffi et al. (2012), as condições de tráfego no segmento em estudo não são homogêneas, e cada faixa de tráfego de uma rodovia possui médias de velocidades, intensidades de fluxo e composições de tráfego diferentes. Portanto, o gerenciamento ativo de tráfego pode oferecer benefícios ao harmonizar as velocidades entre as faixas e assim retardar o aparecimento de congestionamentos, reduzir o número de ultrapassagens e o risco de colisões. 


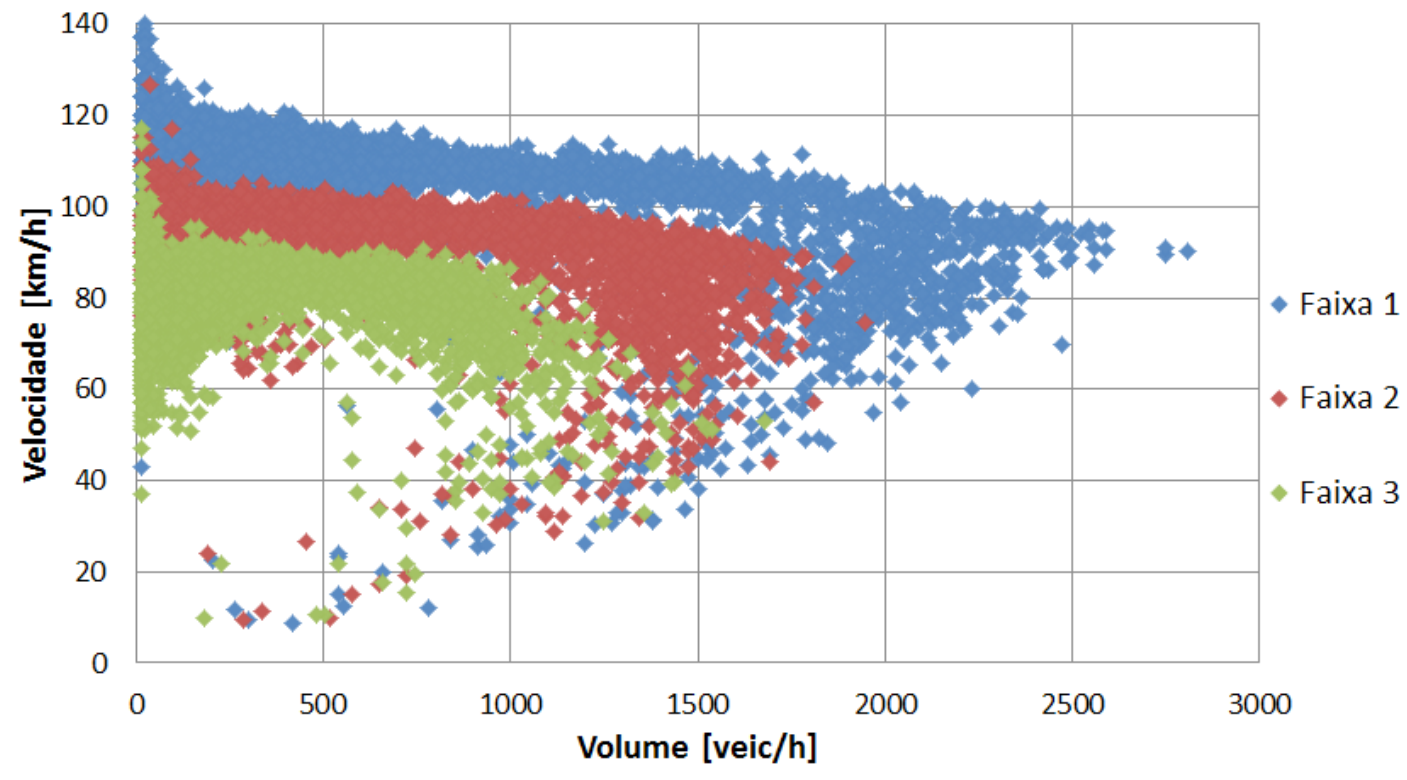

Figura 1: Relação fluxo-velocidade para observações do mês de Janeiro de 2013, km 17, BR-290/RS

Este artigo teve como objetivo a implementação e teste de estratégias de gerenciamento ativo de tráfego para uma autoestrada brasileira, a fim de avaliar e quantificar a eficácia destas estratégias. A estratégia de harmonização da velocidade foi modelada para analisar os efeitos que as reduções dos limites de velocidade têm sobre as correntes de tráfego que passam pelo trecho, em especial nos pontos onde ocorrem quedas bruscas de velocidade, que indicam a formação de congestionamento. A estratégia do uso do acostamento foi modelada para avaliar o impacto que uma faixa adicional de rolamento tem sobre o trecho.

O software de simulação VISSIM foi utilizado para modelagem do trecho em estudo, que corresponde a um segmento da rodovia BR-290/RS, situada no estado do Rio Grande do Sul. Esta rodovia faz a ligação do litoral do estado com a cidade de Porto Alegre e sua região metropolitana, recebendo elevados volumes de tráfego, em particular nos meses de verão. A chave para uma avaliação bem sucedida depende da fidelidade do modelo de simulação utilizado. Portanto, o modelo deve ser calibrado para representar com precisão as condições de tráfego da área modelada.

A Seção 2 deste artigo apresenta uma caracterização do trecho em estudo, trazendo uma análise das condições operacionais da autoestrada em estudo. A Seção 3 descreve a calibração do modelo de simulação. Os cenários de simulação são apresentados na Seção 4. Na Seção 5 é apresentada uma análise dos resultados de simulação e seus impactos no trecho modelado. Conclusões são apresentadas na Seção 6.

\section{CARACTERIZAÇÃO DO TRECHO EM ESTUDO}

Foi selecionado para modelagem um trecho da rodovia BR-290/RS, situada no estado do Rio Grande do Sul, compreendida entre os marcos quilométricos 01 a 10. O trecho em estudo possui uma rampa de acesso no $\mathrm{km} 4$ do trecho. Todo o trecho possui três faixas de rolamento, com exceção de um segmento de 600 metros localizado após a rampa de acesso, que possui quatro faixas. A rodovia possui faixas de rolamento com 3,75 metros de lar- gura cada. O acostamento externo possui 3,5 metros de comprimento. Os limites de velocidade para o trecho em estudo são de $110 \mathrm{~km} / \mathrm{h}$ para veículos leves e $90 \mathrm{~km} / \mathrm{h}$ para veículos pesados.

Uma análise das condições operacionais do trecho em estudo foi realizada a partir de um conjunto de dados coletados na autoestrada através de laços indutivos. Esta análise utilizou dois pontos de coleta de dados, situados a jusante do trecho estudado, nos marcos quilométricos 17 e 19 da BR-290/RS. Esta caracterização do trecho em estudo é importante para compreender o comportamento do tráfego e as condições da via em períodos críticos que precedem a ocorrência de congestionamentos, pois são nestes períodos críticos que as estratégias de gerenciamento ativo de tráfego atuam.

A rodovia recebe elevados fluxos no período do verão e em feriados importantes, onde acontecem as maiores variações de velocidade e intensidade de fluxo. As ocorrências de congestionamentos nestes períodos são, portanto, mais frequentes. A Figura 1 apresenta a relação fluxo-velocidade para uma coleta de dados realizada no $\mathrm{km}$ 17 da rodovia BR-290/RS, para todo o mês de janeiro de 2013. Este ponto da rodovia corresponde a um trecho de reta. A composição de dados está agrupada em intervalos de 5 minutos.

Existe uma diferença significativa entre as velocidades e intensidades de fluxo das três faixas. A primeira faixa da esquerda - Faixa 1, apresenta as maiores velocidades e volumes, enquanto a faixa da direita - Faixa 3, apresenta as menores velocidades e volumes. Um ponto importante é o fato de que a Faixa 3 recebe grande parte do tráfego de caminhões e ônibus, o que acarreta redução da intensidade de fluxo e das velocidades, uma vez que os limites de velocidade são diferentes para veículos leves e pesados. Nesta faixa também predominam o tráfego de veículos leves lentos que trafegam com velocidades muito inferiores às máximas regulamentadas. As porcentagens de veículos pesados na corrente de tráfego, por faixa, são: Faixa $1=1,1 \%$; Faixa $2=10 \%$ e; Faixa $3=23,7 \%$. 


\section{CALIBRAÇÃO DO MODELO}

Os modelos de simulação são especialmente úteis quando as estratégias em análise exigem novas construções ou investimentos onerosos, como é o caso do gerenciamento ativo de tráfego (Park e Qi, 2006).

A calibração é uma etapa fundamental na simulação de tráfego. Nesta etapa, são realizados ajustes nos parâmetros do modelo de forma a refletir com precisão as condições prevalecentes da rede viária. Através de uma revisão de trabalhos de modelagem, Gettman e Head (2003) apontam que o VISSIM possui lógicas de mudanças de faixa e de car following que podem ser configuradas pelo usuário através de parâmetros de calibração. Um bom controle de mudanças de faixa e de car following é essencial para a modelagem deste estudo, pois estes parâmetros possuem grande influência no comportamento do tráfego no trecho em estudo, em períodos de altas intensidades de fluxo.

O processo de calibração envolveu alteração das distribuições de velocidade dos veículos e da distribuição de potência dos automóveis, para compatibilizar com o perfil dos veículos brasileiros. Foram calibrados também parâmetros de comportamento dos condutores. Esta calibração foi divida em duas etapas: análise de sensibilidade - para definir os parâmetros a serem ajustados; e validação dos dados através de uma função objetivo que determina a porcentagem do erro relativo entre os dados modelados e os coletados em campo. Destaca-se que o modelo foi calibrado apenas para dados do caso sem limites de velocidade variáveis. A calibração e posterior validação dos dados foram realizadas através de uma função fitness, que compara a velocidade média dos dados coletados em campo com os dados provenientes do modelo de simulação, respectivamente para os km 19 e 17 da rodovia BR-290/RS. Uma avaliação qualitativa das nuvens de pontos dos gráficos de fluxo-velocidade dos dados coletados e simulados também foi realizada.

\subsection{Análise de sensibilidade dos parâmetros de calibração}

Uma análise de sensibilidade foi conduzida com o objetivo de determinar quais parâmetros de comportamento dos condutores têm impacto sobre a modelagem do trecho em estudo. Foram analisados parâmetros de troca de faixas e de car following para identificar quais parâmetros possuem influência no modelo. Foi então admitida uma gama de valores para cada parâmetro. Uma posterior análise combinatória destes parâmetros, envolvendo 256 combinações, permitiu determinar o melhor conjunto de valores a serem atribuídos a cada um dos parâmetros na calibração.

\subsubsection{Troca de faixas}

A calibração dos parâmetros de troca de faixas foi realizada para melhor representar o comportamento dos condutores, uma vez que no trecho em estudo foi observado um número maior de trocas de faixas e ultrapassagens do que as modeladas com os parâmetros default do VISSIM. O valor do parâmetro "safe distance reduction factor" foi alterado com o objetivo de representar mais adequadamente a agressividade dos condutores durante trocas de faixa.

\subsubsection{Car following}

Através da análise de sensibilidade foi definido que os parâmetros "headway time (CC1)", "following variation (CC2)", "threshold for entering following (CC3)" e "negative and positive following threshold (CC4 e CC5)" possuem influência no desempenho do trecho em estudo e, portanto, deveriam ter seus valores ajustados. $\mathrm{O}$ ajuste destes parâmetros permitiu que o modelo representasse mais adequadamente o comportamento de condutores que trafegam com maior agressividade e mantêm distâncias menores enquanto seguem outro veículo.

\subsection{Obtenção de dados}

A análise da adequação dos parâmetros de calibração foi realizada através de uma comparação dos dados coletados com os dados provenientes do modelo de simulação. Os dados coletados com laços indutivos são provenientes dos km 17 e 19 da rodovia BR-290/RS. Esta comparação é feita através de uma função objetivo, ou fitness, que determina a porcentagem do erro relativo entre as medidas de desempenho obtidas em campo e pelo simulador calibrado. Foram comparadas as velocidades médias dos dados coletados e do modelo calibrado para os km 17 e km 19.

Os laços indutivos posicionados ao longo da rodovia não permitem a obtenção de dados sobre trocas de faixa. Assim, vídeos provenientes de câmaras instaladas na rodovia, nos pontos de coleta dos contadores de tráfego, foram utilizados para calibrar visualmente as trocas de faixa. Para este fim foram contabilizados os números de trocas de faixa por unidade de tempo para três classes de veículos: automóveis, caminhões e ônibus.

Como o objetivo desta modelagem é estudar o comportamento da rodovia e calibrar o modelo para períodos congestionados e que antecedem os congestionamentos, a função objetivo considerou apenas a faixa de velocidades para intensidades de fluxo acima de 4000 veic/hora, uma vez que é a partir destes patamares de volume que acontecem os congestionamentos. A Equação 1 apresenta a função para determinar o valor fitness (erro relativo):

$$
\mathrm{F}(\text { Fitness })=\left(\frac{\mid \mathrm{V} \cdot \mathrm{M} \cdot \text { Coletada }-\mathrm{V} \cdot \mathrm{M}_{\text {Modelada }} \mid}{\mathrm{V} \cdot \mathrm{M} \cdot \text { Coletada }}\right) 100
$$

Em que: $\mathrm{F}$ (Fitness) é a porcentagem do erro relativo;

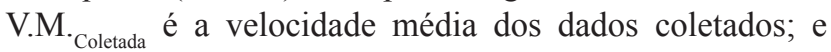
V.M. ${ }_{\text {Modelada }}$ é a velocidade média do modelo de simulação.

\subsection{Comentários sobre o processo de calibração}

Para baixas e médias intensidades de fluxo, os veículos trafegam na maior parte do tempo com bastante liberdade, permitindo aos condutores imprimir velocidades próximas da desejada. Consequentemente, as trocas de faixas acontecem com maior facilidade e os parâmetros de car following passam a ter menor impacto devido às condições confortáveis de tráfego. Para altos volumes, os parâmetros de calibração possuem influência significativa na modelagem, principalmente na capacidade e na representação das trocas de faixa. 
Vel. Média Coletada $(\mathrm{km} / \mathrm{h}) \quad$ Vel. Média Modelada $(\mathrm{km} / \mathrm{h}) \quad$ Fitness $\quad$ Vel. Média $(\mathrm{km} / \mathrm{h}) \quad$ Fitness

\begin{tabular}{llllll}
\hline $\mathbf{k m ~ 1 7}$ & 88,75 & 86,40 & $2,64 \%$ & 82,29 & $7,27 \%$ \\
\hline $\mathbf{k m ~ 1 9}$ & 80,12 & 79,81 & $0,38 \%$ & 72,40 & $9,63 \%$ \\
\hline
\end{tabular}

Como parte do processo de codificação do modelo, foram definidas as distribuições de velocidades desejadas para as diversas categorias de veículos com base nos dados de velocidade coletados em condição de fluxo livre. Esta codificação das distribuições é fundamental para representar de forma satisfatória o trecho em estudo, em especial quando os veículos trafegam em condições confortáveis de tráfego, imprimindo suas velocidades desejadas - períodos estes em que os parâmetros de car following são menos significativos.

Através do cálculo da porcentagem do erro relativo, foi possível determinar o melhor valor fitness para cada ponto de coleta. A Tabela 1 apresenta as velocidades médias e os valores fitness para a combinação de parâmetros considerada calibrada, e os valores fitness para o modelo com os parâmetros default.

Os elevados valores fitness para o modelo de simulação com seus parâmetros default comprovam que a calibração dos parâmetros se faz necessária, para que assim a modelagem possa representar de forma satisfatória as características do trecho em estudo. A Figura 2 apresenta uma comparação entre os dados coletados e o modelado calibrado.

Quando a intensidade de fluxo atinge valores acima de 4000 veic/hora, a variação das médias de velocidade aumenta significativamente. É neste período que ocorrem o início dos congestionamentos, e quando os parâmetros de car following e de troca de faixas têm sua maior influência na modelagem.

$\mathrm{km} 17$

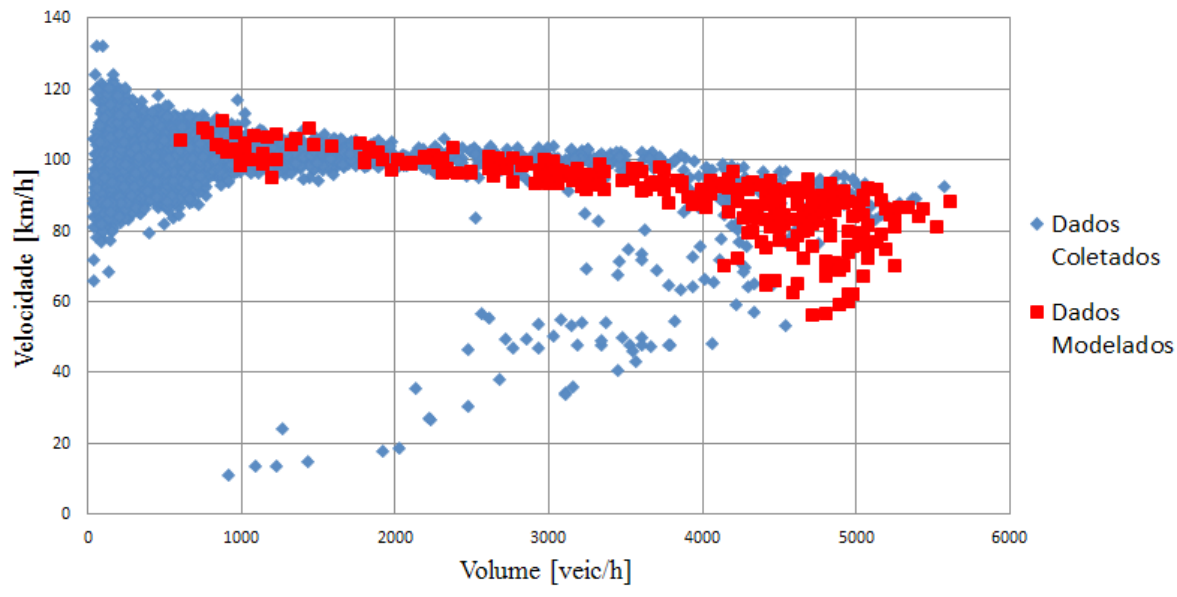

$\mathrm{km} 19$

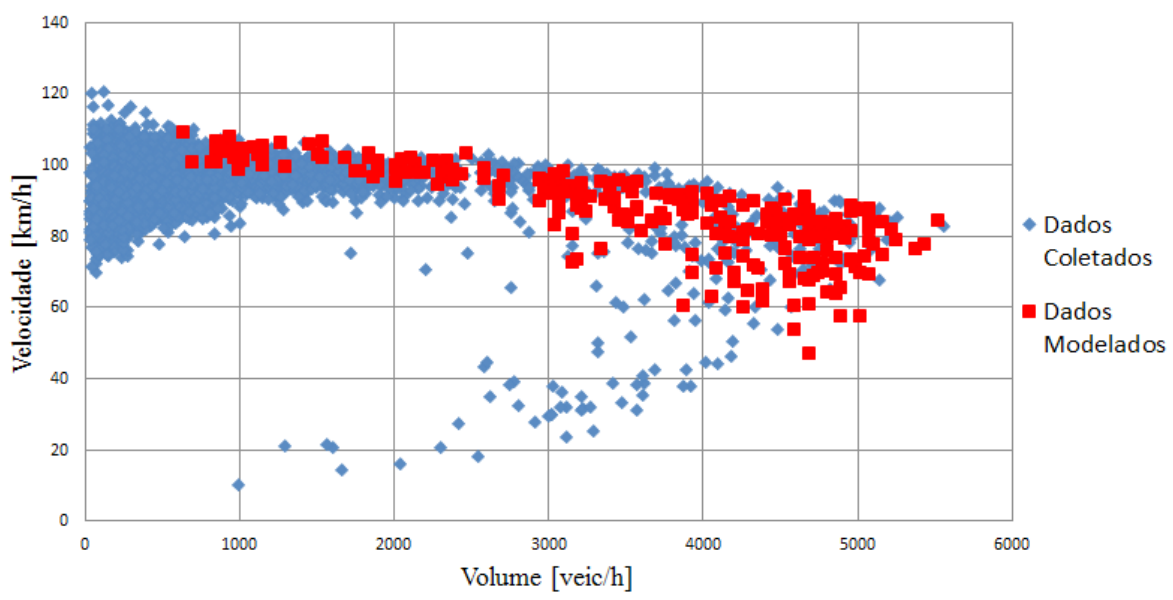

Figura 2: Comparação entre os dados coletados através de laços indutivos e dados do modelo de simulação calibrado, para os km 17 e 19, BR-290/RS 


\section{MODELAGEM DO TRECHO EM ESTUDO}

O VISSIM fornece ferramentas que possibilitam implementar estratégias de gerenciamento ativo de tráfego no modelo de simulação, como o vehicle actuated programming (VAP). O VAP é uma ferramenta de programação que possibilitou a simulação de estratégias de controle de tráfego em resposta às condições do tráfego em tempo real (PTV, 2010).

Foram desenvolvidos três conjuntos de simulações (A, B e C) - cada conjunto com seis replicações. Observouse que, a partir de seis replicações, os valores médios dos parâmetros não apresentavam diferenças significativas. $\mathrm{Na}$ simulação $\mathrm{A}$, o trecho foi modelado sem nenhuma estratégia de gerenciamento ativo de tráfego, a fim de representar o comportamento deste trecho em condições normais de tráfego. Na simulação B, foi modelada apenas a estratégia de harmonização da velocidade, usando limites de velocidade variável (LVV). Na simulação $\mathrm{C}$, foi modelada a harmonização da velocidade em conjunto com o uso temporário do acostamento. O modelo representa 8 horas de simulação, correspondentes ao período de 14:00 a 22:00 horas.

O experimento apresentado envolveu a simulação de um mesmo perfil de demanda de entrada para os 3 cenários simulados. Desta forma, o experimento não possibilita quantificar um provável aumento de escoamento de tráfego, decorrente da implantação das medidas simuladas. As melhorias de desempenho são notadas apenas no aumento da velocidade.

\subsection{Layout do trecho em estudo}

Para a simulação da harmonização da velocidade e do uso temporário do acostamento, foram criados no modelo de simulação onze pórticos. Estes pórticos são responsáveis por indicar aos usuários uma eventual mudança nos limites de velocidade e indicar quando o acostamento está disponível para uso. Em cada um dos onze pórticos há também detectores responsáveis pela coleta de informações de velocidade, volume e ocupação da via. Os pórticos possuem um espaçamento de 800 metros, que é indicado por Jeffrey et. al. (2011) como sendo o espaçamento médio em rodovias onde o gerenciamento ativo de tráfego foi implantado. O segmento que possui quatro faixas de tráfego acaba no ponto onde está localizado o pórtico 5. A Figura 3 apresenta o trecho em estudo modelado no software VISSIM, com os onze pórticos. Estes pórticos são chamados de $\mathrm{P} 1$ a $\mathrm{P} 11$, respectivamente.

O segmento, do pórtico 5 até o final do trecho, foi selecionado para receber a estratégia do uso temporário do acostamento, uma vez que o tráfego adicional do acesso e a redução do número de faixas de quatro para três faixas no pórtico 5 caracterizam um gargalo na via. Este gargalo é o segmento mais crítico do trecho em estudo. Neste ponto a incidência de congestionamentos é maior, e as médias de velocidade reduzem significativamente quando acontecem estes congestionamentos.

\subsection{Algoritmo de controle do gerenciamento ativo de tráfego}

O algoritmo de controle foi implementado através da ferramenta (VAP) do VISSIM. As estratégias de controle usadas para determinar os limites de velocidade variável (LVV) na operação da harmonização da velocidade são fundamentais para o sucesso do funcionamento do sistema. Estas estratégias são aplicadas através de um algoritmo que compara indicadores e limites que acionam a harmonização da velocidade e/ou uso temporário do acostamento. Através das informações provenientes de detectores é possível fazer o controle destas estratégias em tempo real, otimizando o processo (Nezamuddin et al., 2011).

O algoritmo de controle foi projetado para selecionar os limites de velocidade e o uso temporário do acostamento com base em medidas médias de volume, velocidade e ocupação da via durante um período de 2 minutos. Estes limites são selecionados através de informações provenientes dos detectores que estão posicionados em cada um dos onze pórticos. Tais detectores estão posicionados em todas as faixas de tráfego e no acostamento. A ocupação só é medida no acostamento quando este está em uso. Este design incorpora o estado da prática dos primeiros sistemas de gerenciamento ativo de tráfego. Exemplos de algoritmos e seus limites podem ser encontrados em Allaby et al. (2007) e Papageorgiou et al. (2008).

Os valores dos parâmetros para o algoritmo de controle modelado foram selecionados com base em um estudo de caracterização das condições operacionais do trecho em períodos críticos que precedem a ocorrência de congestionamentos. Um limiar de volume de 4000 veículos por hora foi selecionado a partir da análise de dados históricos de sensores da rodovia. Observou-se que este é o volume de tráfego a partir do qual as velocidades médias dos veículos apresentam quedas significativas, que precedem a ocorrência de congestionamentos. Para a ocupação, foi selecionado um limiar de $15 \%$, pois dados revelaram que este limite se aproxima da ocupação crítica, na qual as velocidades

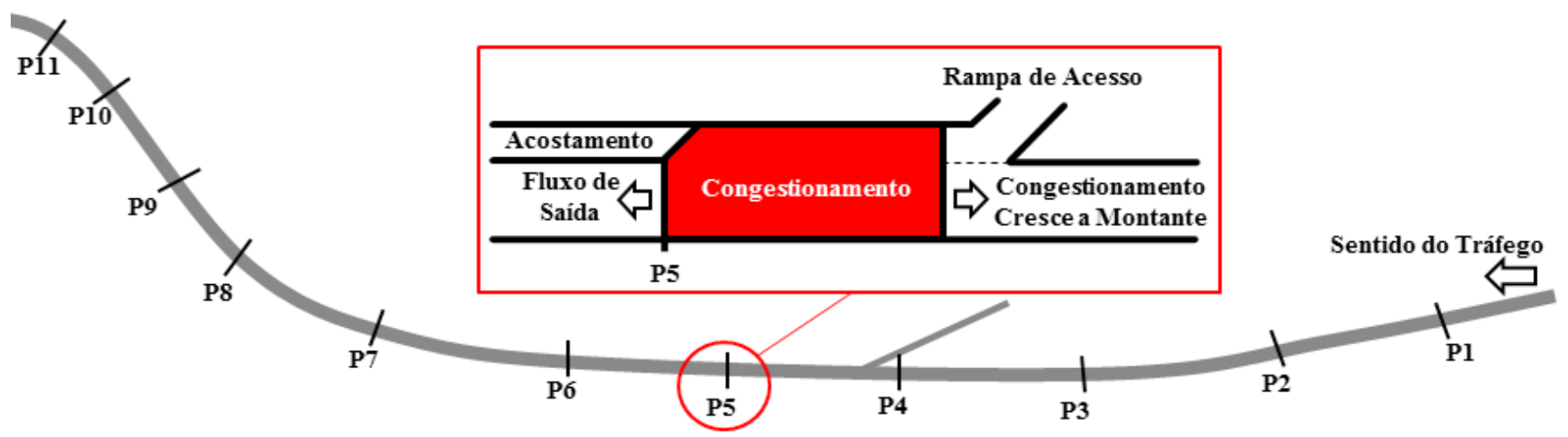

Figura 3: Trecho em estudo 


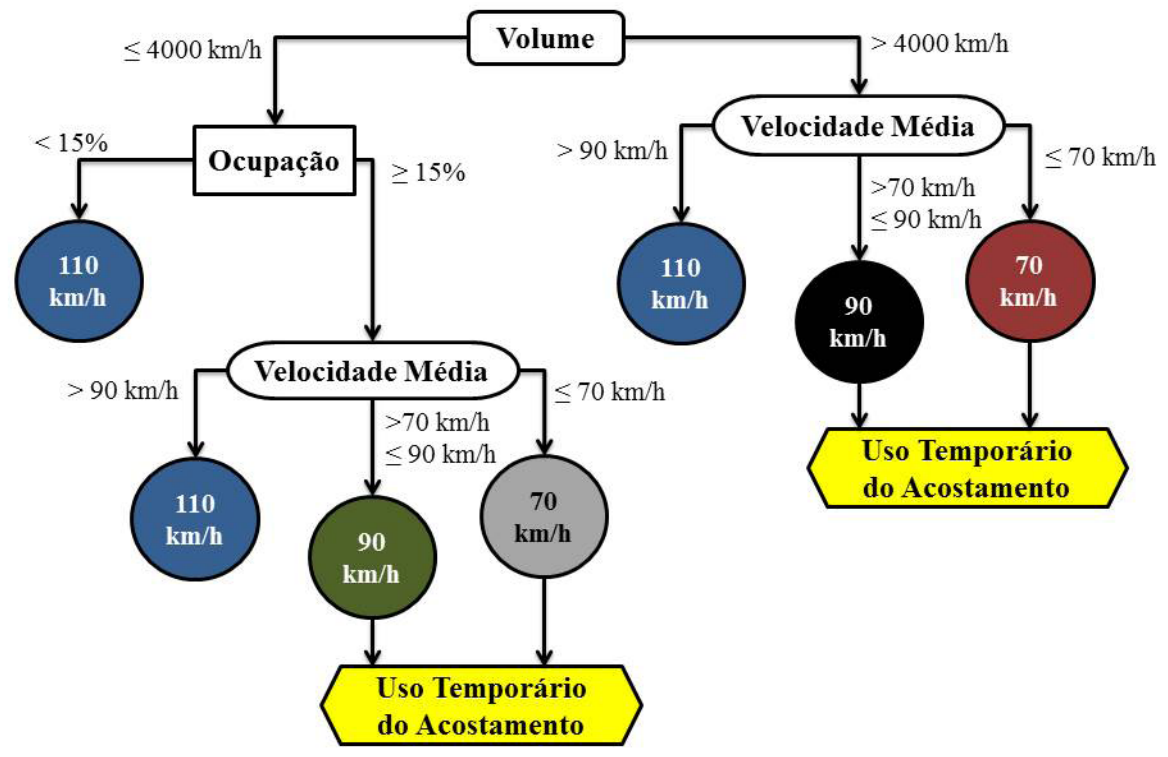

Figura 4 - Fluxograma do algoritmo de controle

médias apresentam quedas mais acentuadas. A ocupação é determinada pelo percentual de tempo que o contador de tráfego está ocupado. Maiores volumes de veículos e baixas velocidades contribuem para elevar a ocupação. Foram usados três limites de velocidade na modelagem. A velocidade limite da via $(110 \mathrm{~km} / \mathrm{h})$; a primeira redução de velocidade $(90 \mathrm{~km} / \mathrm{h})$; e a segunda redução de velocidade $(70 \mathrm{~km} / \mathrm{h})$.

Uma vez que o sistema de gerenciamento ativo de tráfego é ativado no pórtico onde há a detecção dos limiares do algoritmo de controle, as velocidades indicadas nos pórticos a montante são determinadas com base em uma zona de atuação e uma zona de transição, descritas a seguir:

- Zona de atuação - Harmonização da velocidade e/ou uso temporário do acostamento são ativados no pórtico de detecção e nos dois pórticos mais próximos a montante. Se o limite de velocidade ativado for reduzido de 110 para $90 \mathrm{~km} / \mathrm{h}$, os pórticos a montante exibem os mesmos limites de velocidade do pórtico de detecção;

- Zona de transição - Se o limite de velocidade ativado for reduzido de 110 para $70 \mathrm{~km} / \mathrm{h}$, o último pórtico da zona de atuação (segundo pórtico a montante) deve exibir o limite de velocidade de $90 \mathrm{~km} / \mathrm{h}$, para proporcionar uma transição gradual de redução de velocidade para os condutores.

O primeiro passo do algoritmo de controle consiste na leitura dos detectores. As médias das leituras relativas a cada dois minutos são comparadas com os limiares de volume, ocupação e velocidades definidos no estudo de caracterização do trecho, para determinar se o sistema de gerenciamento ativo deve entrar em operação. O sistema é desativado quando detectores indicam que a corrente de tráfego atinge volume menor que 4000 veic/hora e ocupação menor que $15 \%$.

Quando as condições de tráfego encontram-se próximas ao limiar de ativação, pode-se observar uma oscilação indesejável no sistema, com ativações e desativações frequentes. Este estado é referido na literatura como chaveamento. Em situações reais é importante conceber mecanis- mos de proteção contra o chaveamento (Allaby et al. 2007; Papageorgiou et al. 2008). Este algoritmo, em particular, não prevê mecanismos deste tipo, uma vez que as condições simuladas apresentam um perfil de demanda conhecido e controlado. A Figura 4 apresenta o fluxograma do algoritmo de controle usado na modelagem, quando as estratégias de LVV e uso temporário do acostamento são usadas em conjunto.

A Figura 5 apresenta a relação fluxo-velocidade das médias de todas as faixas da rodovia para a coleta de dados realizada no km 17, feita no mês de janeiro de 2013. Esta figura aponta o conjunto de dados onde o sistema de gerenciamento ativo de tráfego estaria ativado, de acordo com os limiares do algoritmo de controle.

As linhas vermelhas indicam os dois limiares de velocidade que ativam o sistema, e a linha verde o limiar de volume que ativa o sistema. Os pontos azuis indicam o estado da rodovia em que o sistema de gerenciamento ativo de tráfego estaria desativado, e operando com o limite de velocidade da via de $110 \mathrm{~km} / \mathrm{h}$. Os pontos pretos, marrons, verdes e cinzas indicam o estado da rodovia em que o sistema estaria ativado. Os pontos cinza e verdes indicam quando a rodovia tem um volume inferior a $4000 \mathrm{veic} / \mathrm{h}$, mas possui uma ocupação superior a $15 \%$. As cores usadas nesta figura correspondem às cores usadas no fluxograma da Figura 4.

\section{ANÁLISE dOS RESULTADOS DE SIMULAÇÃO}

A partir dos resultados de simulação é possível analisar os efeitos que as estratégias de gerenciamento ativo de tráfego têm sobre o trecho em estudo. Ao dividir a modelagem em três simulações (A - sem controle, $\mathrm{B}$ - com a estratégia de harmonização da velocidade e $\mathrm{C}$ - harmonização da velocidade e uso temporário do acostamento), é possível analisar separadamente os efeitos de apenas a estratégia de LVV, e das estratégias implantadas em conjunto. Os impactos da modelagem nas velocidades médias, no tempo de viagem e nas trocas de faixa foram analisados. Os dados apresentados nesta seção correspondem à média das seis replicações realizadas. 


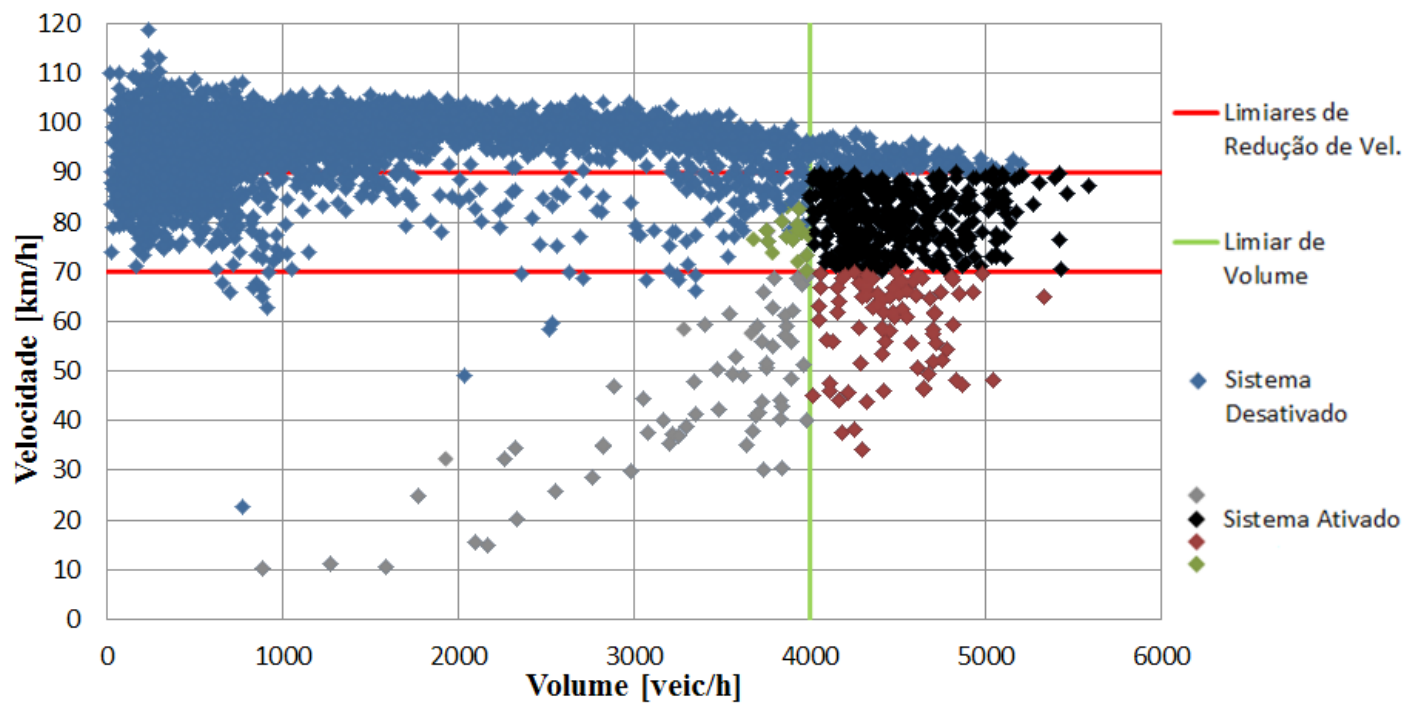

Figura 5: Relação fluxo-velocidade da rodovia, km 19, BR-290/RS

Pórtico 5
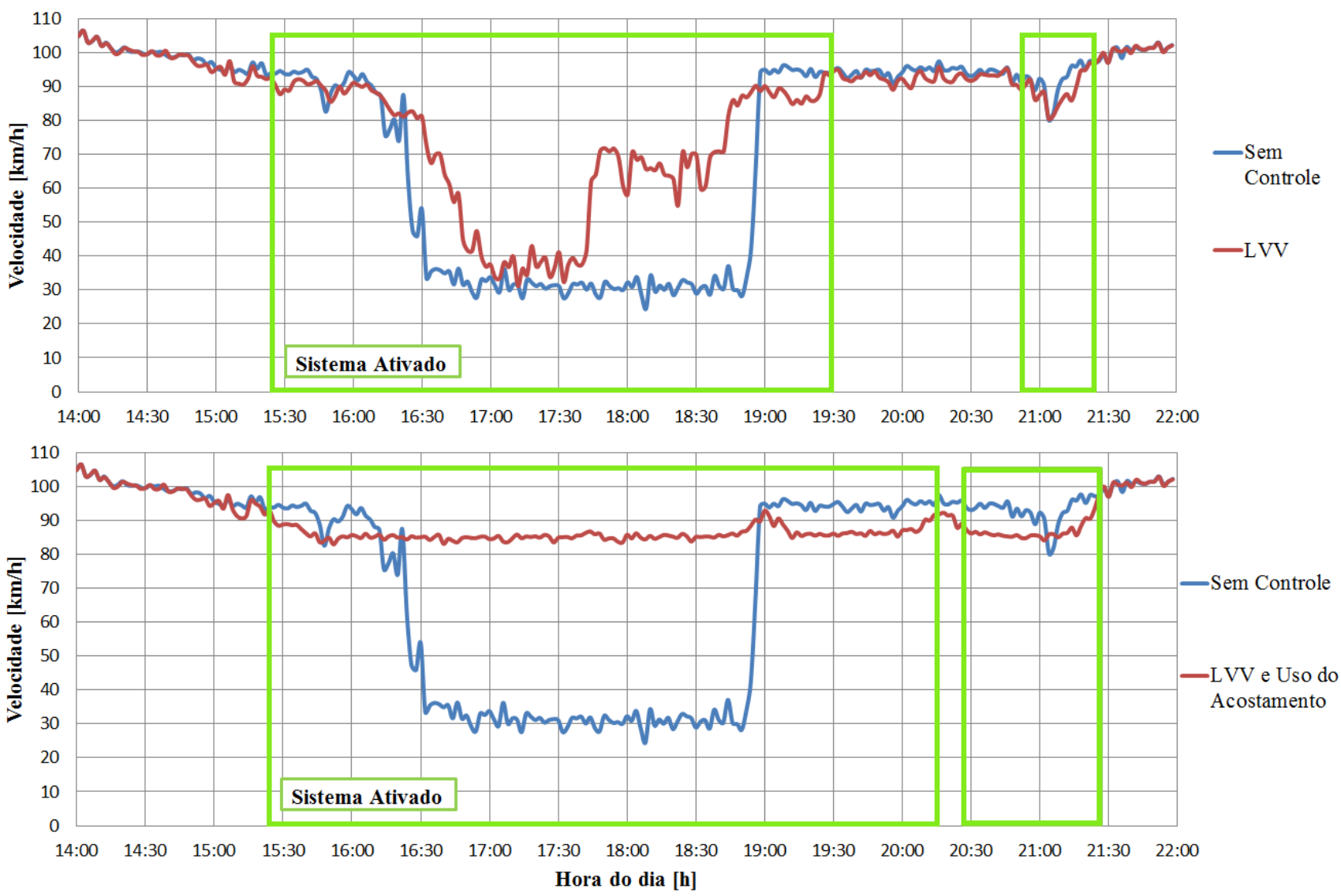

Figura 6: Comparação das velocidades médias e volume para o pórtico 5

\subsection{Impacto nas velocidades médias}

O pórtico 5 é o trecho que apresenta as menores médias de velocidades quando a via atinge sua capacidade. Neste ponto as velocidades caem devido à perturbação gerada pelo acesso e pela redução de quatro para três faixas. Portanto, é neste ponto que as estratégias de gerenciamento ativo de tráfego têm seu maior impacto no tráfego. A Figura 6 apresenta os resultados das simulações A, B e
C para o pórtico 5. Cada ponto corresponde à média dos veículos a cada 2 minutos.

Pode-se notar na Figura 6 que quando os limites de velocidade variável (LVV) passam a ser empregados, há um retardo no aparecimento de quedas bruscas de velocidade. É possível notar também que com o uso de LVV, o período em que a via permanece em estado de velocidades muito reduzidas é menor. Com o uso de LVV é possível ter um maior controle quando o gargalo no pórtico 5 está ati- 
Tempo de Viagem

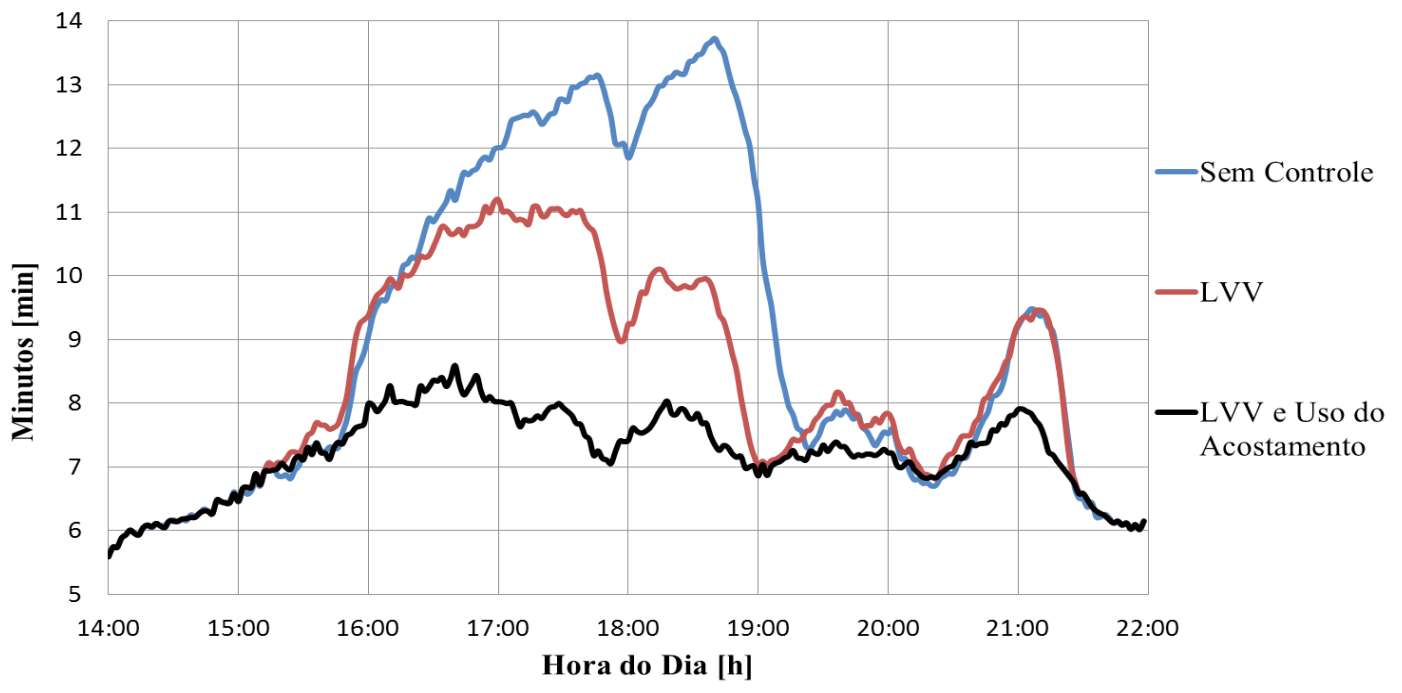

Figura 7: Comparação dos tempos de viagem

vo. Nota-se que, a partir das 17:30, há um aumento das velocidades. Uma provável justificativa para este fenômeno pode ser creditada às consequências da harmonização das velocidades a montante deste gargalo. A harmonização de velocidade acarreta uma redução nos headways entre os veículos, nas trocas de faixa e ultrapassagens, reduzindo a probabilidade de conflitos e gerando uma corrente de tráfego mais controlada.

Usando a estratégia de harmonização da velocidade (LVV), é possível apenas reduzir os efeitos da brusca queda de velocidades observada durante o congestionamento. Porém, ao acrescentar a estratégia do uso temporário do acostamento (LVV e Uso do Acostamento) neste sistema de gerenciamento ativo de tráfego, os congestionamentos são completamente eliminados, com as velocidades médias se mantendo em patamares elevados.

O uso temporário do acostamento fornece uma faixa adicional de rolamento em períodos congestionados, eliminando o gargalo - ponto de redução no número de faixas no pórtico 5. Para se obter efeitos positivos no ponto onde há quedas bruscas de velocidade, o sistema deve ser ativado a montante, para harmonizar o comportamento do tráfego antes do ponto de início do colapso do fluxo. Assim é possível ter um fluxo mais regular e com menos conflitos ao se aproximar do ponto de colapso. Quando a rodovia está operando na sua capacidade, nota-se que com o uso das estratégias de gerenciamento ativo de tráfego o fluxo apresenta uma menor oscilação, se mantendo em níveis mais elevados.

Com o uso conjunto das duas estratégias, nota-se que o sistema permanece ativo por um período de tempo mais longo. Isto se deve à hipótese simulada em que os veículos que fazem uso do acostamento trafegam com as mesmas velocidades dos veículos que estão na Faixa 3. Levando em conta as particularidades das rodovias brasileiras apontadas anteriormente sobre a diferença das médias de velocidade para cada faixa da rodovia (Faixa 3 é a mais lenta), ao fazer uso do acostamento a média de velocidade da rodovia reduz, mantendo o sistema ativo por um período maior.

\subsection{Impacto nos tempos de viagem}

Um dos benefícios dos sistemas de gerenciamento ativo de tráfego, apontados por Sisiopiku et al. (2009) e Mirshahi et al. (2007), é o aumento da confiabilidade no tempo de viagem. Como apontam Fontaine e Miller (2012), diversas rodovias com implantação do gerenciamento ativo de tráfego tiveram redução nos tempos de viagem, bem como uma redução na variação destes tempos de viagem.

Os impactos dos tempos de viagem modelados foram medidos comparando, a cada 2 minutos, as médias dos tempos de viagem dos veículos que percorreram todo o trecho, que possui $10 \mathrm{~km}$ de extensão. A Figura 7 apresenta uma comparação dos tempos de viagem para as simulações $A$, $\mathrm{B}$ e $\mathrm{C}$, que representam o modelo sem nenhum controle simulação A; com controle de harmonização da velocidade (LVV) - simulação B e; com LVV e uso temporário do acostamento - simulação C.

Pode-se notar uma significativa redução nos tempos de viagem quando as estratégias de gerenciamento ativo de tráfego são empregadas. No período entre 16:00 e 20:00 horas, período este em que a rodovia estava operando com altos volumes de tráfego, é possível perceber a maior diferença entre os tempos de viagem para as três simulações.

No período das 21:00 horas, o volume da rodovia chega a sua capacidade, porém por um curto intervalo de tempo. Quando a rodovia opera na sua capacidade por um período de tempo pequeno, as velocidades não sofrem uma grande variação, e não há o aparecimento de quedas bruscas de velocidade. Em consequência, os tempos de viagem do sistema com apenas LVV permanecem os mesmos do modelo sem nenhum controle. A harmonização da velocidade só tem impacto nos tempos de viagem quando ocorrem grandes quedas de velocidade. Usando as duas estratégias LVV e uso do acostamento, nota-se uma grande redução na variação do tempo de viagem, o que representa um tempo de viagem mais confiável. A Tabela 2 apresenta, para o período entre 16:00 e 20:00 horas, uma comparação entre os picos máximos e mínimos; a faixa de variação nos tempos de viagem; e o tempo médio de viagem. 
Tabela 2: Comparações dos tempos de viagem para a rodovia operando com altos volumes

16:00 a 20:00 horas

\begin{tabular}{llll}
\hline Tempo de Viagem & Sem Controle (min) & LVV (min) & LVV e Uso do Acostamento (min) \\
\hline Máximo & $13: 43$ & $11: 11$ & $08: 35$ \\
\hline Mínimo & $07: 17$ & $07: 01$ & $06: 51$ \\
\hline Faixa de Variação & $06: 26$ & $04: 10$ & $01: 44$ \\
\hline Tempo Médio & $11: 51$ & $09: 49$ & $07: 40$ \\
\hline
\end{tabular}

Com a rodovia operando com a estratégia de LVV, o tempo médio de viagem reduz-se $17 \%$, e a variação do tempo de viagem reduz $35 \%$. Já com a rodovia operando com as duas estratégias - LVV e uso do acostamento, o tempo médio de viagem reduz $35 \%$, e a variação reduz $73 \%$. Estes resultados apontam que o gerenciamento ativo de tráfego tem um grande impacto positivo nos tempos de viagem do trecho modelado, reduzindo significativamente o pico máximo, a variação e o tempo médio das viagens.

\subsection{Impacto nas trocas de faixa}

Outro benefício dos sistemas de gerenciamento ativo de tráfego é o aumento da segurança no tráfego. Uma das formas de mensurar o aumento da segurança é através da avaliação do número de trocas de faixa que acontecem durante uma viagem. Ao utilizar a estratégia de harmonização da velocidade, as trocas de faixa diminuem, pois uma vez que os veículos trafegam com uma menor variação de velocidades, acontecem menos ultrapassagens e consequentemente menos trocas de faixa. Ao reduzir o número de trocas de faixa, a probabilidade de ocorrer incidentes também reduz (Mirshahi et al., 2007).

$\mathrm{Na}$ autoestrada em estudo o número de trocas de faixa dos veículos é maior do que naturalmente representado através de parâmetros default dos modelos de lane changing. Com isso, o simulador foi calibrado a partir de análises de vídeos para que as trocas de faixa no modelo representem a realidade do trecho em estudo.

Os impactos das trocas de faixa foram medidos comparando, a cada 2 minutos, o número de trocas de faixa que ocorreram ao longo dos $10 \mathrm{~km}$ do trecho modelado. A Figura 8 apresenta uma comparação das trocas de faixa para as simulações modeladas. Com o uso da estratégia de LVV, o número de trocas de faixa reduziu 30\%. Já com as estratégias de LVV e uso do acostamento, o número de trocas de faixa reduziu $43 \%$.

Mesmo com uma faixa de rolamento adicional com o uso temporário do acostamento, o número de trocas de faixa é menor. Isto se deve ao fato de que usando as duas estratégias em conjunto, as quedas bruscas de velocidade que acontecem nas imediações do pórtico 5 são eliminadas, como ilustra a Figura 6. Eliminando os efeitos destas quedas de velocidade, os veículos trafegam livremente neste trecho, evitando os conflitos que poderiam levar os veículos a trocarem de faixa devido às reduções de velocidade.

\section{CONCLUSÕES}

Este artigo apresentou a modelagem de estratégias de gerenciamento ativo de tráfego para um trecho de uma autoestrada brasileira. As estratégias modeladas foram a de harmonização da velocidade e a de uso temporário do acostamento. A modelagem demonstrou que o gerenciamento ativo de tráfego proposto tem impactos positivos na operação do tráfego.

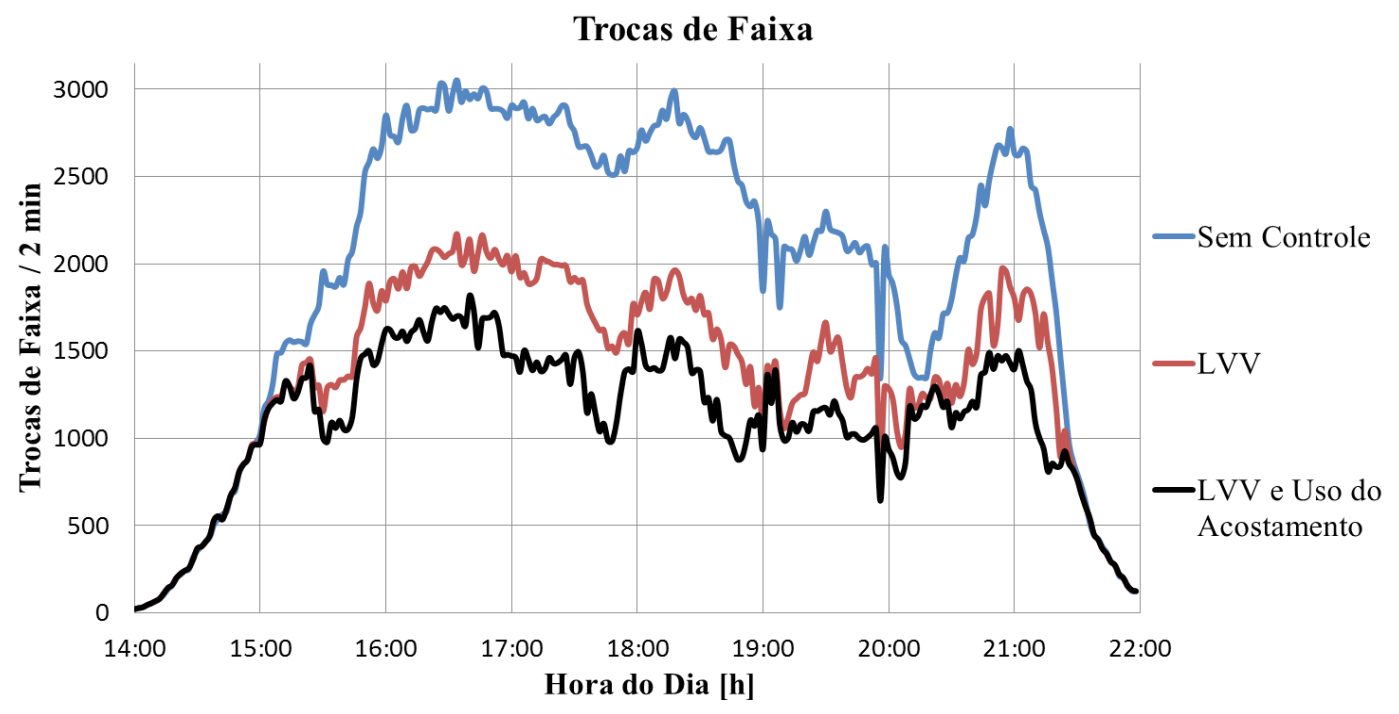

Figura 8: Comparação das trocas de faixa 
O uso do sistema de gerenciamento ativo de tráfego proposto reduziu os tempos médios das viagens e a variação dos tempos destas viagens, e reduziu as trocas de faixa, o que implica em uma redução dos conflitos e da probabilidade de ocorrer incidentes. Com o uso do sistema houve também redução do tempo em que a via opera com baixas médias de velocidade, aumentando a eficiência do trecho.

A modelagem indicou os seguintes resultados para apenas a estratégia de harmonização da velocidade (LVV): redução visual dos headways; redução de $17 \%$ no tempo médio das viagens; redução de $35 \%$ na variação do tempo de viagem e; redução de $30 \%$ nas trocas de faixa.

$\mathrm{O}$ uso conjunto das estratégias de gerenciamento ativo de tráfego leva a um melhor aperfeiçoamento das condições operacionais da via. Para este uso conjunto de harmonização da velocidade e uso temporário do acostamento a modelagem indicou os seguintes resultados: redução visual dos headways; redução de $35 \%$ no tempo médio das viagens; redução de $73 \%$ na variação do tempo de viagem e; redução de $43 \%$ nas trocas de faixa. Como o experimento envolveu a simulação de um mesmo perfil de demanda de entrada para todos os cenários, não foi possível quantificar um provável aumento de escoamento de tráfego, decorrente da implantação das medidas simuladas.

O sucesso das estratégias de gerenciamento ativo de tráfego depende principalmente da compreensão e do comportamento dos usuários quanto aos métodos de aplicação destas estratégias. No universo de modelagem, o comportamento dos veículos é pré-determinado pelo simulador de tráfego, e muitas vezes pode não representar a variação de comportamento apresentada pelos usuários num cenário real de tráfego. $\mathrm{O}$ mesmo pode ser afirmado quanto à compreensão das estratégias de controle empregadas no simulador. Com isso, numa implantação do gerenciamento ativo de tráfego em rodovias, deve-se levar em consideração questões como a compreensão dos usuários perante novas estratégias de controle. Através de uma boa compreensão por parte dos usuários é possível minimizar a variação de comportamento ao implantar estratégias inovadoras.

\section{AGRADECIMENTOS}

Os autores agradecem o apoio financeiro da CAPES, através de uma bolsa de doutorado. Os autores também agradecem o apoio da concessionária CONCEPA e da ANTT, que disponibilizaram os dados de tráfego.

\section{REFERÊNCIAS}

Allaby, P., Hellinga, B., e Bullock, M. (2007). Variable speed limits: safety and Operational Impacts of a Candidate Control Strategy for Freeway Applications. IEEE Transactions on Intelligent Transportation Systems, 8(4), 671-680. DOI: 10.1109/ TITS.2007.908562

Caleffi, F., Y. Moisan, H. B. B. Cybis, F. D. Michel e F. Hirsh (2012). Simulação de alternativas operacionais de um segmento especial de autoestrada através do software VISSIM. CONGRESSO DE PESQUISA E ENSINO EM TRANSPORTES,26,.2012. Anais... Belo Horizonte: ANPET, v. 1, p. 36-47.
Carlson, R. C., Papamichail, I., Papageorgiou, M., e Messmer, A. (2010). Optimal motorway traffic flow control involving variable speed limits and ramp metering. Transportation Science, 44(2), 238-253. DOI: 10.1287/trsc.1090.0314

Mirshahi, M., Obenberger, J., Fuhs, C. A., Howard, C. E., Krammes, R. A., Kuhn, B. T., Mayhew, R. M., Moore, M. A., Sahebjam, K., Stone, C. J., e Yung, J. L. (2007). Active traffic management: the next step in congestion management. Federal Highway Administration, Report no. fhwa-pl-07-012, Washington D.C.

Jeffrey C. Jones, Knopp, M. C., Fitzpatrick, K., Doctor, M. A., Howard, C. E., Laragan, G. M., Rosenow, J. A., Struve, B. A., Thrasher, B. A., e Young, E. G. (2011). Freeway geometric design for active traffic management in Europe. Federal Highway Administration, Report No. FHWA-PL-11-004, Washington D.C.

Fontaine, M. D. e J. S. Miller (2012). Planning for active traffic management in virginia: International Best Practices and Implementation Strategies. Virginia Center for Transportation Inovation and reaserch, Final Report VCTIR 13-R1, USA.

Geistefeldt, J. (2013) Impact of Variable Speed Limits on Motorway Level of Service. International Journal of Intelligent Transportation Systems Research, 12(2), 61-69. DOI: 10.1007/ s13177-013-0072-x

Gettman, D., e Head, L. (2003). Surrogate safety measures from traffic simulation models. Transportation Research Record: Journal of the Transportation Research Board, 1840(1), 104115. DOI: $10.3141 / 1840-12$

Nezamuddin, N., N. Jiang, T. Zhang, S. T. Waller e D. Sun (2011). Traffic operations and safety benefits of active traffic strategies on TxDOT Freeways. Federal Highways Administration, Publication Number: FHWA/TX-12/0-6576-1, USA.

Papageorgiou, M., Kosmatopoulos, E., e Papamichail, I. (2008). Effects of variable speed limits on motorway traffic flow. Transportation Research Record: Journal of the Transportation Research Board, 2047(-1), 37-48. DOI: 10.3141/2047-05

Park, B. B., e Qi, H. M. (2006) Microscopic simulation model calibration and validation for freeway work zone network - a case study of VISSIM. 2006. IEEE Intelligent Transportation Systems Conference, 1471-1476. DOI: 10.1109/ITSC.2006.1707431

PTV (2010) Vissim user manual: v.5.30. Karlsruhe, Alemanha.

Sisiopiku, V. P., Sullivan, A., e Fadel, G. (2009). Implementing active traffic management strategies in the U.S. University transportation center for Alabama, Department of Management and Safety in Transportation Systems.Report no. 08206, USA.

Vadde, R.; D. Sun, J. O. Sai, M. A. Faruqi e P. T. Leelani (2012). A simulation study of using active traffic management stategies on congested freeways. Journal of Modern Transportation, v. 20, n. 3, p. 178-184. DOI: 10.1007/BF03325796 